\title{
L'alta atmosfera e una nuova proprietà del plasma (ionosfera) $(*)$
}

\author{
M. (UTOLO $\left({ }^{* *}\right)$ \\ Ricevuto il 18 Maggio 1963
}

Riassuxto. - Si premettono alcune notizie sulla costituzione dell'atmosfera e della ionosfera (Fig. 1), e sulla variazione della temperatura con l'altezza (Fin. 2), secondo le conoscenze piu recenti.

Dopo aver accennato alla presenza nell'alta atmosfera delle correnti dinamo, si descrive la ragione del comportamento non-lineare del plasma ionosferico.

Segue la descrizione dell'effetto di girointerazione e la dimostrazione teorica dell'esistenza delle curve di risonanza dromedaria e bactrian.

Data la spiceata non-linearita della parte bassa della ionosfera, lautore penso che il plasma poteva comportarsi come un " detector " o "rivelatore", separando la bassa frequenza dalla portante di un'onda modulata quando questa si propaga attraverso il plasma.

Gli esperimenti fatti tra il 1953 ed il 1962 confermano l'ipotesi avanzata dallautore, e cioe che la ionosfera per la sua non linearita da luogo ad un effetto di rivelazione.

A mezzo dell'efietto di rivelazione e stato possibile imprimere l'impulso di un'onda disturbatrice su di un'onda ricercata e generare cosi l'effetto Lussemburgo con risonanza (Girointerazione).

Il nuovo effetto e la tecnica descritta dovrebbero permettere di studiare il plasma interplanetario invece che sempre con satelliti, anche solo con radioonde trasmesse da terra, risparmiando così notevoli somme finanziarie.

SUMMARY. - We give first some news on the constitution of the atmosphere and ionosphere (Fig. 1), and on the variation of the temperature in relation with the height (Fig. 2) as by the most recent knowledges.

(*) Nota presentata al $2^{\circ}$ Congresso Internazionale Tecnico Scientifico dello spazio. Roma, 19-23 Gingno 1962.

(**) Centro Studi di Fisica dello spazio. - Istituto di Fisica Tecnica (Ingegneria) ed Istituto di Fisica (Architettura) dell'Universita di Napoli. 
We report about the presence of the dymamo-currents in the high atmosphere and of a description of the reason why the ionospheric plasma behaves in a non-linear way.

We give a description of the grrointeraction effect and a theoretical demonstration about the existence of the dromedary and hactrian resonance curves.

Due to the remarkable non-linearity of the lower part of the ionosphere, the author thought that the plasma may hehave as a "detector" separating the low frequency from the carrier of a molulated wave.

This paper refers to the experiments earried out between 1953 and 1962 which confirm that a detection effect does occur.

By means of the detection effect it has been possible to impress the pulse of the disturbing wave on the wanted wave.

The new effect and technique should permit us to study the interplanetary plasma by transmitting radio waves from earth. This would mean saving a great deal of money because artificial satellites wouldn`t bo always neederl.

Il plasma (gas ionizzato) rhe rircondat la terra fla i 60 ed i $500 \mathrm{~km}$ di altezza dalla superficio terrestre e che ha il nome di ionosfera e stato sudrliviso in tre regioni denominate rispettivamente: $F$ (che di giomo si scincle negli strati $\left.F_{1}+F_{2}\right), E$ a $D$.

Mentre la regione $F$ si trova al disopra dei $240 \mathrm{~km}$ erl ha una densita elettronica $N$ (numero di elettroni per ('m³) che varia in maniera complicata duante la notte, lo strato $F_{1}$ ha $N \sim 10^{5}$ a circa $160 \mathrm{~km}$ ed $F_{2}$ ha $N \sim 10^{\circ}$ a cirmal $260 \mathrm{~km}$.

La densita elettronica della regione $E$ valia naturalnente dal giomo alla notte. Di giorno $N$ ha un massimo ( $~ 10^{5}$ elettroni $/\left(\cdot \mathrm{m}^{3}\right)$ a circa $100 \mathrm{~km}$. Questa altezza però varia con la latitudine, la stagione, l'oma diurna e il ciclo delle macohie solari. Dumente la notte $N$ diminuisce marcatamente sino a ridursi a poche decine o centinaia ri elettroni liberi per conº Al contrario dello strato $F_{2}$ che ha uno spessore maggiope di jo km, $E$ e speesso rire'a $10 \mathrm{~km}$.

Dai $60 \mathrm{~km}$ di altezza agli 80 si trova la regione $D$ la cui densita elettronica e compresa tra $10^{3}$ e $10^{1}$ elettroni/(mon. Dopo il tramonto del sole $D$ scompare totalnente.

La composizione dellatmosfera rimane quasi la stessa dal livello del mare sino a grandi altezze. Essa e costituita cone a noto, principalmente da azoto $\left(N_{2}\right)$ e ossigeno $\left(\mathrm{O}_{2}\right)$ biatomici.

Intorno ai $100 \mathrm{~km}$ di altezza l'ossigeno da biatomico diventa monoatomice. Questo cambiamento, il quale produce una grande modificazione delle proprieta chiniche a assorbenti di luce di $O$, e doruto al fenomeno 
della fotodissociazione generato dalla luce solare. Per quanto riguarda invece il cambiamento di $\mathrm{X}$ da biatomico a monoatomico, il processo e poco chiaro. Sembra che esso sia doruto alla tendenza delle molecole piu pesanti a separarsi al disopra dei $160 \mathrm{~km}$ dalle altre per effetto della gravita che naturalmente e piu intensa per le molecole piu pesanti.

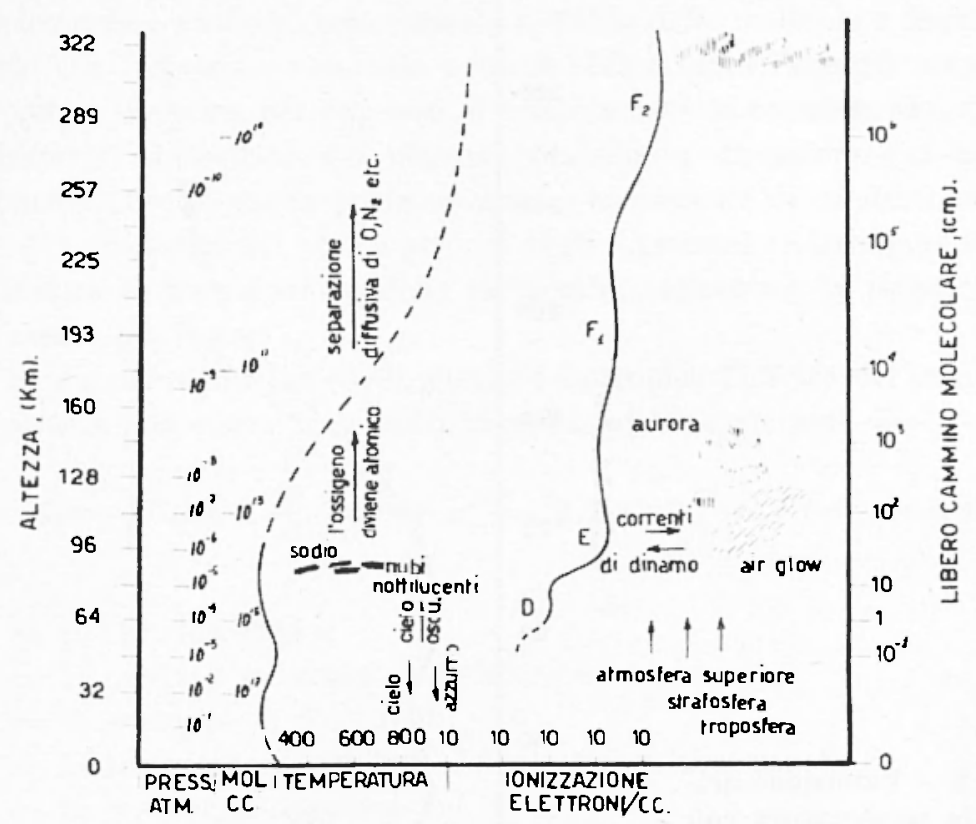

Figr. I - Libero cammino molecolare. (em).

Come si vede dalla Fig. 1 la temperatula diminuisce con l'altezza raggiungendo un prino minimo a circa $24 \mathrm{~km}$ per poi risalire sino ad avere un massino a $50 \mathrm{~km}$. Dojo una nuova dininuzione la temperatura comincia a salire sensibilmente dai $100 \mathrm{~km}$ in su con una legge non ancora ben chiara tanto che sono state a vanzate due ipotesi rappresentate dalle due linee tratteggrate di Fig. 2. In ogni caso la cosa certa e che la temperatura raggriunge alti valori a grandi altezze.

Il grafico di Figr. 1 indica le pressioni, il numero di nolecole per ce e i ammini liberi degli elettroni nell'aria alle varie altezze $\left.{ }^{1}\right)$.

1. - CORRENT DINAMO F COMPORTMENTO YOS LINGARE DEL PLASMA.

Le regioni che qui a noi interessano per quello che diremo in seguito sono la $E$ e la 7 . Como si vede in Fig. 1 la regione $E$ a la sede di quel 
sistema di correnti responsabili della fluttuazione del campo magnetico terrestre alla superficie terrestre. Queste correnti sono chiamate dinamo e sono generate dall'azione gravitazionale esercitata sugli elettroni liberi da parte del Sole e della Luna. Esse sono chiamate dinamo perche si

Fig. 2 - Variazione del-

la temperatura con l'altezza.

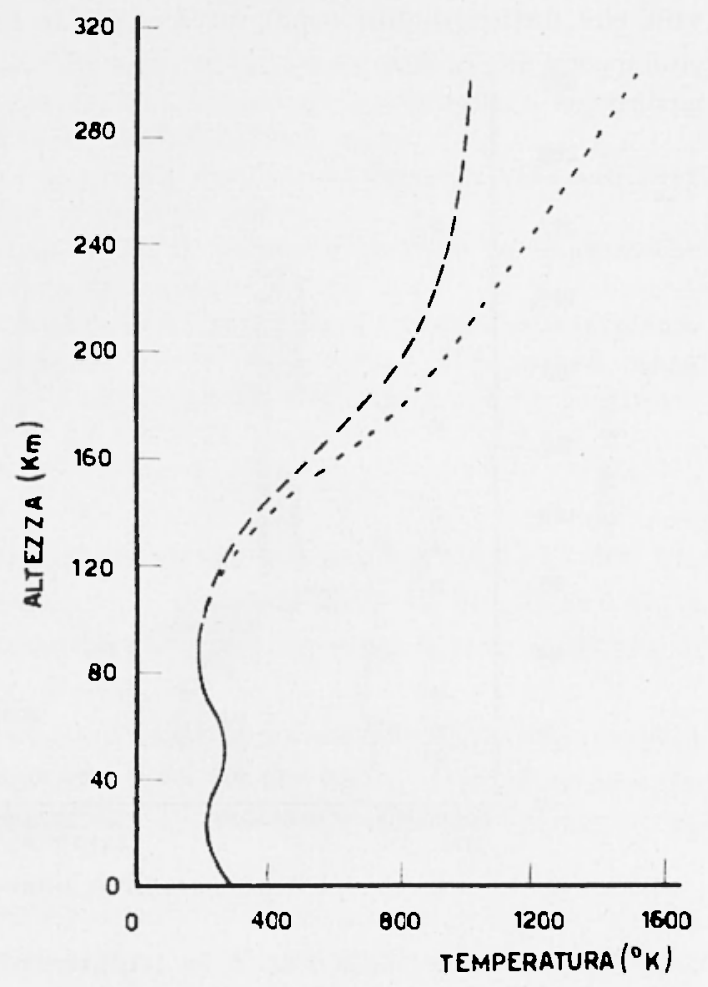

producono nella stessa guisa delle correnti elettriche generate da una dinamo. Se noi ricordiamo che questa macehina e formata da una bobina avvolta su un toro di ferro, chiamata rotore, che gira entro un campo magnetico prodotto da un magnete, si ha che nel caso della ionosfera, il magnete e sostituito dal campo magnetico terrestre e la bobina dalla ionosfera la quale, come il rotore, e conduttrice per effetto degli elettroni liberi esistenti in essa.

Le correnti elettriche si generano perche gli elettroni, per effetto di marea, si muovono attraverso le linee di forza del campo magnetico terrestres.

Oltre alla presenza del sistema di correnti elettriche di cui ora si e parlato, la regione $E$, al pari di quella 7 , ha un particolare comportamento nella propagazione delle radioonde. 
Dato che il numero degli elettroni e in sostanza molto esigno (di notte $\mathrm{N}=100$ elettroni $/ \mathrm{cm}^{3}$ o anche meno) e il cammino medio e notevole, l'elettrone tra un urto e l'altro con le molecole neutre e gli ioni ha il tempo di acquistare molta energia dal campo elettrico della radioonda che attraversa la regione $E(0 \quad D)$ della ionosfera. Ma poiche la massa dell'elettrone e molto piccola rispetto a quella delle molecole e degli ioni, accade che l'elettrone non cerle a quest'ultimi tutta l'energia acquisita dal campo elettrico per cui esso si riscalda con la consequenza che la permittivita dielettrio'a del plasma comincia a dipendere dal campo elettrico $E$. Segue che la polarizzazione e la corrente di conduzione non sono piu proporzionali ad $E$, per eui tutti i processi elettrodinamici, in particolare la propagazione delle radioonde, acquistano la proprieta di non essere piu lineari.

Se noi immaginiamo che il plasma è isotropico l'effetto del campo $E$ sul plasma puo essere trascurato in prima approssimazione se

$E \ll E _ { p } = \longdiv { 3 K T \frac { m } { e ^ { 2 } } G ( \omega ^ { 2 } + v _ { o } ) } = 4 . 2 . 1 0 ^ { - 1 0 } \longdiv { G T ( \omega ^ { 2 } + v _ { 0 } ^ { 2 } ) } \mathrm { r } / \mathrm { cm }$ dove:

$E_{p}=$ il " plasma field ";

e, $m=$ la carica e la massa dell'elettrone;

$K=$ la costante di Boltzman;

$T=$ la temperatuma assoluta del plasma in assenza di $E$ :

(1) = la frequenza angolare del campo;

$a$ = la perdita media di energia subita da un elettrone contro le particelle pesanti;

$v_{0}=$ la frequenza di collisione quando non c'e il campo $E$.

Soltanto se $E \gtrsim E_{p}$ o meglio se $E \gg E_{p}$ le proprieta del plasma sono sostanzialmente cambiate. Se $(1)^{-} \ll v_{o}=$ si ha che nella regione $E$ : $E_{p}=\sim 10^{-\mathbf{5}}\left(\nu_{o} \sim 10^{5}, T \simeq 300^{\circ}, G \simeq 10^{-3}\right)$ mentre nella corona solare $E_{p} \sim 10^{-i} \mathrm{~V} / \mathrm{cm}\left(\gamma_{o} \sim 10, T \sim 10^{6}, G=1 / 918\right)\left(^{2}\right)$.

\section{2. - LA GIROINTERAZIONE.}

Come e noto, l'effetto Lussemburgo consiste nel trasferimento (nella ionosfera) di una piccola parte della modulazione di una radioonda di frequenza media e della potenza di un centinaio di kW (detta onda disturbatrice) su di un'altra onda di frequenza completamente diver'sa dalla prima, non molulata e di potenza inferiore (chiamata ricercata). In generale il percento della modulazione trasferita, detta anche modulazione 
parassita o " cross-modulation ", e dell"ordine dell:1 o $2 \%$ " con una potenza della stazione disturbatrice dell'ordine del centinaio di $\mathrm{kW}$.

Tel 1937 il Prof. V. A. Bailey ha scoperto teoriamente che se la frequenza della stazione disturbatrice invece di essere una frequenza qualsiasi e uguale all'incirca alla frequenza giromagnetica (o frequenza ciclotronica) locale della ionosfera si sarebbe doruto avere, per effetto di risonanza, un'esaltazione del fenomeno della interazione per cui il percento della modulazione trasferita sarebbe dovuto aumentare di 4-10 volte rispetto al percento di morlulazione parassita che si ottiene nel semplice effetto Jussemburgo $\left(^{3}\right)$.

A questo nuovo fenomeno preristo soltanto teoricamente il Bailey ha rato il nome di intelazione con risonamza o "girointerazione".

Lo studio di questa teoria eseguito da me nel 1942 mi convinse che qualom il fenomeno veramente fosse esistito esso sarebbe stato opportunamente arloperato per potere misurare direttamente la frequenza giromagnetica locale della ionosfera e per conseguenza il campo magnetico terrestre nella alta atmosfera.

In altri termini il fenomeno della girointerazione avrebbe permesso la verifica della teoria magnetoionica formulata nel 1925 da Appleton, e cioe l'esistenza nella ionosfera di una frequenza giromagnetica, teoria che sino al 1946 e stata confermata in parte soltanto con esperienze di laboratorio da parte di vari ricereatori fra i quali in primo piano V. De Pace e pii diffusamente G. Torlesco.

Il piano delle ricerche e la descrizione della difficile organizzazione sono illustrate in $\left(^{4}\right)$. I risultati della esperienza eseguita nel Marzo $1946\left(^{5}\right)$ e alla quale parteciparono vari importanti Enti militari, Vaticani e universitari sono i seguenti:

1) Per la prima rolta e stato possibile mettere in luce l'esistenza della risonanza come aveva previsto teoricamente il Bailey.

2) Per effetto della risonanza e stato possibile ottenere il fenomeno con una potenza inferiore al kilowatt larldove l'effetto Lussemburgo era stato ottenuto all'estero sempre con potenze dell'ordine del rentinaio di $\mathrm{k} W$.

3) È stato possibile per la prima rolta dimostrare sperimentalmente l'esistenza nella ionosfera di una frequenza giromagnetica così come era stata definita matematicamente dalla teoria mannetoionica.

4) Fu possibile mostrare una variazione regolare con l'ora notturna della frequenza di risonanza (da non confondere con la frequenza giromagnetica), variazione non sospettata teoricamente a che sembra dovuta alla variazione della densita elettronicar dell'alta atmosfera. 
Intanto la teoria della girointerazione prevede che. in fumzione della penetrazione nello strato $E$ dell'onda ricercata, vi possono essere due curve di risonanza e, precisamente, o una curva con due massimi simmetrici rispetto alla girofrequenza oppure una curva con un sol massimo poco discosto dalla girofrequenza $\left({ }^{3}\right)$.

Nel Marzo del 1949 organizzai una nuora campagna di esperienze col proposito di fare il tentativo di redere se era possibile deteminare sperimentalmente la curva di risonanza a due massimi prevista dalla teoria della girointerazione. Lorganizzazione della nuova campagna richiese molto lavoro particolarmente per preparare la stazione perturbatrice.

Durante il Giugno-Luglio dello stesso amno furono eseguite 12 notti di esperienze in cui fu possibile costruire sperimentalmente la curva a due massimi che il Bailey avera previsto nel 1938.

In questo ciclo sperimentale fu osservata l'influenza delle macchie solari sul fenomeno della girointerazione.

Dopo la verifica sperimentale dellesistenza di una curva a due massimi restava la comprova sperimentale dellesistenza anche della curva con un sol massimo. Allo scopo di fare specifica verifica e stata organizzata nel 1950 una nuova campagna di esperienze. Servendomi sempre dello stesso metodo di misura e delle stesse stazioni trasmittenti e ricerenti adoperate nel 1949, furono eseguite, tra il Maggio e l'agosto del 1950,17 notti di esperienze.

In questo faticoso laroro solo due rolte si ottenne la curva a un massimo. Durante una notte fu possibile, adoperando la consueta frequenza di Taranto di $475 \mathrm{kc} / \mathrm{s}$ e operando la variazione della portante di Firenze II intorno alla grirofrequenza, ottenere una chiama curva di risonanza ad un sol massimo. E stato notato che in quel giomo l'attivita solare era bassa. In un'altma notte, invere, si ottemne la culva di risonanza a due massimi con la frequenza di Tamanto di $175 \mathrm{kc} / \mathrm{s}$. Spostata questa frequenza di una trentina di chilocicti si passo dalla curra a due massimi alla curva a un massimo $\left(^{6}\right)$.

Concludendo si deduce che il nostro contributo a consistito:

1) Dimostrazione dell'esistenza del fenomeno della risonanza nell'interazione delle radioonde nella ionosfera mediante potenze inferiori al kW adoperando il metodo della variazione della frequenza portante intomo alla frequenza ciclotronica (girofrequenza) e successivamente il metodo degli impulsi.

2) Terifica sperimentale dell'esistenza della curva di risonanza a due massimi simmetrici rispetto alla girofrequenza (bactrian-curve). 
3) Dimostrazione dell'esistenza della curva di risonanza col massimo all’incirca sulla girofrequenza ottenuta facendo variare la penetrazione dell'onda nello strato $E$ della ionosfera.

it interessante notare che il Prof. V. A. Bailey per confermare le curve da me osservate dovette attendere che la densita elettronica dello strato $E$ subisse una diminuzione col progredire dell'ora notturna, e cio per ottenere la sola ('urva a un massimo (7). In tale maniera e chiaro che egli non segui il mio metodo, cioe non fece variare la penetrazione dell'onda ricercata nella ionosfera.

\section{3. - BRENI CONSLDERMZIONI TEORICHE.}

Si tratta ora di vedere come si possono giustificare le due curve di risonanza e cioe quella a due massimi simmetrici rispetto alla frequenza giromagnetica $\Omega_{H} / 2 \pi$ e quella con un massimo all'incirca su $\Omega_{H} / 2 \pi$.

Se si suppone che il gas ionizzato sia immerso in un campo magnetico costante (per esempio: il campo magnetico terrestre) il moto di un elettrone libero fra due urti consecutivi con le molecole neutre, sotto l'azione di un campo elettrico alternativo e dato dalla seguente equazione di V. A. Bailey:

$$
a \frac{d v}{d t}+\frac{1}{2} a\left(t\left(v^{2}-v_{0}^{2}\right)=W_{o}(t)\right.
$$

dove

$$
\begin{aligned}
v \quad= & \text { frequenza di collisione degli elettroni; } \\
v_{o}= & \text { valore di } \nu \text { all'equilibrio temico; } \\
a \quad= & \text { prodotto della massa dell'elettrone per il quadrato del am- } \\
& \text { mino libero medio; } \\
G \quad & \text { energia relativa media perduta in una collisione; } \\
W_{o}(t)= & \text { ralore medio del lavoro fatto dal campo elettrico su un elet- } \\
& \text { trone durante due urti consecutivi di questi con le molecole } \\
& \text { neutre. }
\end{aligned}
$$

Il valore medio di $W_{0}$ e dato dalla seguente espressione:

$$
\overline{W_{0}}=\frac{2 k}{N \nu} u
$$

dove $l e$ e il coefficiente di assorbimento del mezzo, $u$ il valore medio del flusso di energia dell'onda attraverso una superficie di area unitaria normale alla direzione di propagazione, y la frequenza di collisione. 
Con successivi sviluppi si ha:

$$
\bar{u}=\tau \iota_{s} Z^{2} \frac{\frac{1}{2} \operatorname{sen}^{2} \psi^{\prime}}{\vartheta^{2}+\sigma^{-}-\vartheta^{2} p^{\prime} \sigma^{2} / \Omega_{H}}
$$

dove $\tau=e^{2} / m ; Z=$ valore efficace del campo elettrico dell'onda nel punto di incidenza; $\varphi=$ angolo tra il vettore campo elettrico dellonda e il ampo magnetico telrestre: $\nu=$ frequenza di collisione degli elettroni, secondo la distribuzione di Martrn

$$
=1,1 \cdot 10^{6} \exp \left[-y \cdot 10^{-4}\right] ;
$$

dove:

$y=$ alteza verticale nello strato al disopra del livello 0 supposto a $90 \mathrm{~km}$ di altezza;

$$
\sigma=\omega-\Omega
$$

dove:

(1) $/ 2 \pi=$ frequenza dell'onda;

$\Omega_{H} /-\pi=$ frequenza grimamatica locale;

$$
p^{\prime}=\frac{\operatorname{tg}^{2} \theta+2 \cot \alpha^{2} \varphi}{\operatorname{ton}^{2} \theta+2}-\frac{\tau N\left(1+\cos ^{2} \theta\right)}{2 \varepsilon_{o} \eta^{2}}
$$

dove:

$N=$ numero di elettroni per $\left(\mathrm{Am}^{3}\right.$;

$\theta$ = angolo tra la direzione di propagazione dell'onda ed il campo magnetico terrestre;

$\varepsilon_{0}=$ costante dielettrica del vuoto;

as e definita dalla relazione:

$$
\log a_{s}=-2 \int_{0}^{s} k \cdot d_{s}=-0,0532\left(\cos i_{0}+\sec \left(i_{0}\right) \int_{0}^{s} \frac{\pi t}{t^{2}+\sigma^{2}} d y\right.
$$

dove:

$$
\text { li e stato gia definito e } \operatorname{tg} a=\cos \theta \text {. }
$$

Quando un'onda elettromagnetica attraversa la ionosfera, si scinde, come e noto, in due onde dette rispettivamente onda ordinaria e onda straordinaria. Particolare interesse suscita il calcolo w per i due tipi di onda, in funzione dei parametri $N, v e l$ secondo la [3]. In uno dei lavori di Bailey e in mo dell'autore $\left({ }^{6}\right)$, sono state riportate due tabelle in cui sono stati dati i valori di $\bar{w}$ rispettivamente per l'onda st laordinaria e per quella ordinaria. 
Dallesame delle due tabelle, rhe per brevita qui non si riportono, scaturise ehe mentre per l'onda ordinaria $\bar{w}$ cresce con la frequenza dell'oncla senza alcumche di particolare, per quella satraordinalia vo e piceolo per frequenze inferiori o superiori a $Q_{H} / 2 \pi$ mentre che per la frequenza $\Omega / 2 \pi=\sim \Omega_{H} / 2 \pi \bar{w}$ jresenta un valore massimo straordinario e cioe si produce mu notevole grado di risonanza.

Dalla [2] si ha che woy - $2 k u$. Bailey ha chiamato il prodotto Nvero "indice di interazione " perche esso rappresenta la media energia assorbita dagli elettroni per unita di volume e per secondo. Poiehe $\bar{w}$ ha un valore massimo quando l'onda ha la sua frequenza proprio uguale alla grirofrequenza $\Omega_{H} 2 \pi$, lassorbimento della componente straordinaria in tale ipotesi a cosi grande pispetto all'onda ordinaria che leftetto di quest'ultima e quasi tmasemabile.

Ar una data altezza ncllo strato $E$, $\bar{w}$ e data dall'intensita del campo elettrico dellonda a quell'altezzal, e dalla frazione di energia dellonda assorbita. Come l'onda peneta nello strato, orvero aumenta l'altezza, la frazione di energia assorbita resesce rapirlamente mentre l'intensita dell'oncla stessa comincia a diminuile.

Per una data frequenza dellonda, l'indice d'interazione $N \bar{v}$ o lagrgriunge un massimo a una determinata altezza. L'assorbimento della componente straoldinaria ha un massimo piuttosto preciso su la girof requenza per cui l'assorbimento dell'onda su la girofrecuenza e molto piu grande di quello di un'onda la cui frequenza e di pochi percento minore o pii grande della girofrequenza. Di qui scaturisce che l'indice d'interazione ha il suo massimo valore di penetrazione molto piiz piccolo per un'onda la cui frequenza è uguale o meglio quasi uguale alla gimofrequenza di quella di un'onda la cui frequenza e lontana dalla gimofrequenza.

A livelli non molto lontani dal bordo inferiore dello strato $E$ (90 lim) l'iudice d'interazione es massimo per la girofrequenza, mentre a livelli piu alti lindice puo essere minore per la girofrequenza (per il fatto che l'onda e diventata molto debole) a molto glande per le frequenze che sono un po' pii piccole o piu grandi della grirofrequenza stessa.

In questo modo si spiega la ragione dell'esistenza di ma curva di lisomanza a due massimi a a mo massimo.

La Fig. 3 rappresenta le curve teoriche calcolate da Bailey per il cielo di Londla, nell'ipotesi di un campo magnetico rerticale, di una potenza irradiata di $10 \mathrm{k}$ W e di un angolo di incidenza sul livello $y=0$ di $45^{\circ}$.

Dall'esame del diagramma si rileva che per penetrazione dell'onda nello strato $E$ compreso tra 2 e 4 lim, si ha un solo massimo dellat funzione di Nve, molto prossimo alla girofrequenza; non appena la penetrazione 
supera il valore di t, $5 \mathrm{~km}$, si hamno due massimi distinti della funzione. Giova aggiungere che, con l'ulteriore ammento della penetrazione il calcolo mostra ohe i due punti di massimo si hamno in corrispondenza di frequenze sempre piu lontane dalla frequenza giromagnetica e la curva tende ad appiattirsi sino al punto che la risonanza praticamente scompare.

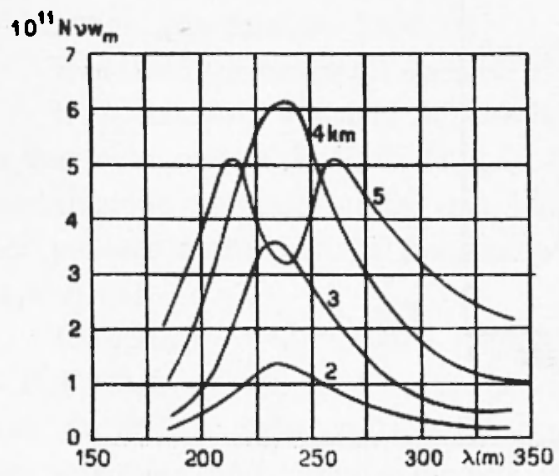

Figr. 3 - Diagramma della funzione $f v \bar{w}_{o}-f(z)$ calcolata per la frequenza giromagnetica pertinente al cielo di Lomblra; parametro delle curve e la profondita $y$ di penetrazione dellomda rirercata nello sitrato $E$.

Se ora si sceglie opportunamente la frequenza portante di uncaltra onda (onda ricercata) di frequenza completamente differente da quella sinora considerata, e che noi qui chiamiamo onda disturbatrice, e si dispongono le stazioni in modo che la distanza tra l'enettitore di questa e il posto di ricezione abbia un determinato valore, si ha che il punto di riflessione dellonda ricereata puo capitare proprio nella zona della ionosfera dove l'indiee d'interazione $N v \bar{c}_{0}$ della disturbatrie si comporta nel modo suindicato. Operando in tal modo si ha che una parte non trascumabile della modulazione della onda disturbatrice (nell'ipotesi che essa sia modılata) si trasferisce sull'onda ricereata che, per semplicita, consideriamo non modulata. Si genera in questo modo la girointerazione o effetto Lusssemburgo con risonanzal.

Il perento di modulazione (cross-modulation) $y$ impresso dalla disturbatrice su la ricereata e fornito dalla relazione:

$$
M=c \int_{0}^{s} X_{n} w_{s}, s,
$$

dove:

$s=$ tratio pereorso dallonda ricercalta nella ionosfera;

$c=$ funzione, quasi costante, dei parametri geometrici della propalgazione dellonda ricercata e della frequenza e profondita di modulazione dell'onda perturbatrice. 
Te Figg. 4 e 5 rappresentano rispettivamente le curve di risonanza a due massimi e a un massimo ottenute sperimentalmente dall'autore il 13 Luglio 1949 ed il 7 Giugno $1950\left(^{6}\right)$.
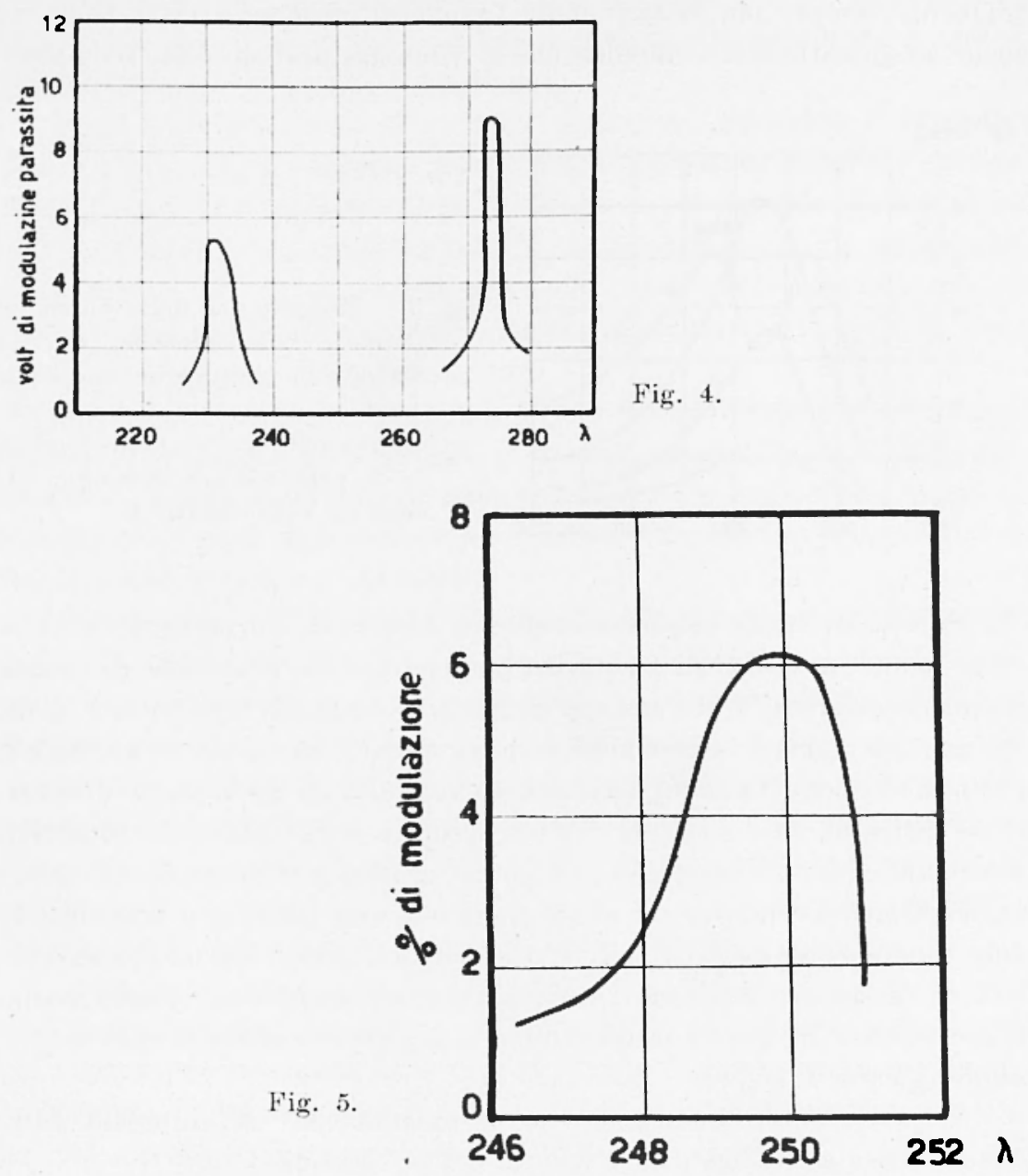

4. - UNA NUOVA pRoprieta DEL PIASMA (10NOSYERA).

Come conseguenza di queste esperienze e del comportamento non lineare del bordo inferiore dello strato $E$, come risulta dai lavori di L. G. Huxley, B. Van der Pol, D. Graffi, M. Cutolo, V. L. Ginzburg, A. V. Gurevich, I. MI. Vilenskij e di altri ricercatori, e da ritenersi che il plasma, per la sua non linearita, possa comportarsi come un rivelatore, separando 
la bassa frequenza dalla portante di un'onda morlulata. Conseguentemente, c'e da aspettarsi che irradiando verso la ionosfera un'onda V.H.F.. modulata con frequenza uguale alla girofrequenza locale, l'inviluppo dell'onda debba avere delle conseguenze sul plasma.

Per verificare questa previsione is stata escogitata, fin dal 1953, una opportuna tecnica descritta in un brevetto richiesto negli Stati Uniti d'America alla fine del $1957\left(^{8}\right)$.

Risultati sperimentali decisivi si sono ottenuti nel 1959 e 1960.

L'esperimento consiste nell'irradiare verso la ionosfera, quasi lungo la verticale, onde V.H.F. da 50 a $75 \mathrm{MI} / \mathrm{s}$ modulate con una frequenza di modulazione variabile entro una banda limitata tra 1000 e $1250 \mathrm{kc} / \mathrm{s}$ per passare attraverso la frequenza ciclotronica, o girofrequenza (1200 $\mathrm{k}(\mathrm{s} / \mathrm{s}$ cir(a)

L'apparato trasmittente emette treni di impulsi rettangolari di V.H.F. della durata di 350 o piu $\mu$ s. Il modulatore consiste di un oscillatore di RF in onda continua, la cui radiofrequenza (1000-1300 kc/s) ron un opportuno circuito modula il trasmettitore V.H.F. La potenza irradiata dal trasmettitore V.H.F. e di circa $80 \mathrm{~kW}$ di picco mentre il percento di modulazione e di $10 \% \%$.

Il sistema aereo e del tipo Yagi con alimentazione incrociata a due piani e con 8 elementi in tutto. Il guadagno e pertanto di 10-12 dB.

Per verificare se, conformemente alle nostre previsioni, l'inviluppo fosse stato capace di agire sul plasma facendo per es. variare la frequenza di collisione del gas ionizzato e quindi modificare il coefficiente di assorbimento del mezzo, si e ricorsi alla stessa tecnica atta a mettere in evidenza l'effetto Lussemburgo ron risonanza (girointerazione).

Poiche gli esperimenti sono stati fatti di giorno, si sono adoperate le tecniche da noi usate nel 1947 e $1948\left({ }^{9}\right)$. Mentre il trasmettitore V.H.F. era situato in Napoli, un altro trasmettitore era locato in Nola (Napoli-Nola: km 25) ed emetteva frequenze portanti tra 1, 7 e $2,2 \mathrm{Mc} / \mathrm{s}$ per avere la riflessione delle onde intorno ai $90 \mathrm{~km}$. La stazione ricevente era sita a Brusciano che dista da Nola km 9 e 16 da Napoli. L'aereo di Nola era un dipolo. Il sistema di aereo di Brusciano ridnceva notevolmente l'intensita del raggio superficiale di Nola. La stazione di Nola emetteva la sua portante senza modulazione in onda continua. Ogni 15 o 20 minuti emetteva le onde a impulsi in modo da controllare l'altezza di riflessione. Non appena si areva la riflessione, a $90 \mathrm{~km}$ o meno, il trasmettitore V.H.F. di Sapoli cominciava a lavorure. Il suo sistema aereo direttivo faceva un piccolo angolo con la verticale in modo da concentrare il fascio nel cielo Nola-Brusciano intorno ai $90 \mathrm{~km}$ di altezza.. 
La presenza di ostacoli e colline tra Napoli e Brusciano faceva si che l'onda V.H.F. non si propagava allatto rerso Brusciano. Opportuni controlli hanno permesso di assodare che la radiofrequenza di $1 \mathrm{Mc} / \mathrm{s}$, modulante l'onda V.H.F., non era ricevuta nemmeno a 1,t km dal trasmettitore.

Non appena l'onda ricercata (Nola) si riflettera, e rio acoudera intorno ai $90 \mathrm{~km}$ di altezza, e il trasmettitore V.H.F. cominciava a funzionare, a avenuto spesso che nel ricevitore sito a Brusciano a acrordato sull'onda di Nola $(1.7-2,2 \mathrm{Mc} / \mathrm{s})$ si percepiva distintamente l'impulso irrudiato da Napoli.

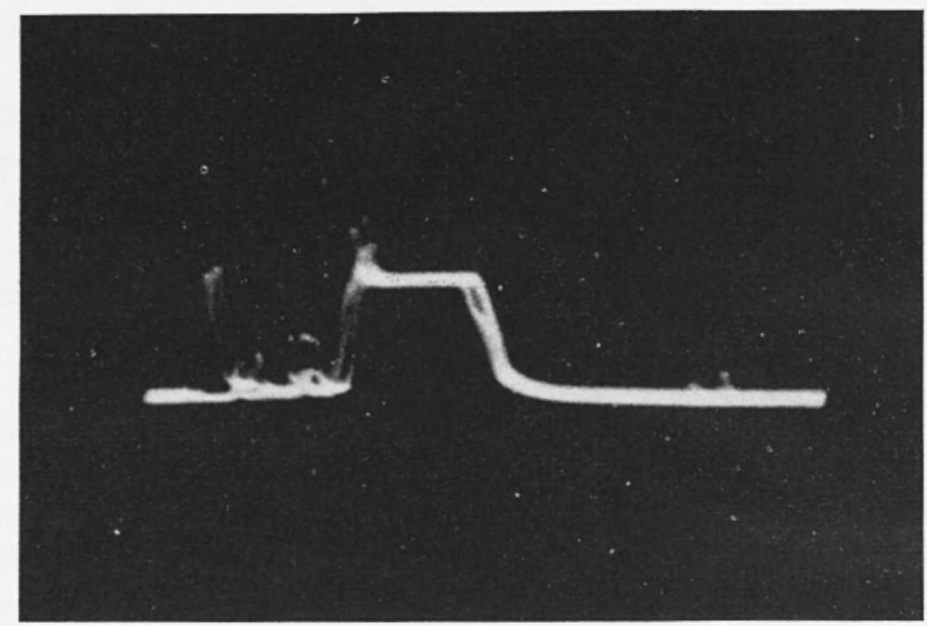

Fig. 6 - Rappresenta limpulso a bassa frequenza impresso dallomba V.H.F. sullonda irradiata dalla stazione di Nola.

Nella serie di esperienze decisive eseguite nel Diembre del 1959 e nel Lugglio del 1960, la modulazione impressa nella ionosfera dal V.H.F. sull'onda di Nola i stata, talvolta, anche del $5-10 \%$.

La Fig. 6 mostra l'impulso a B.F. impresso sull'ouda di Vola datla frequenza modulante della portante V.F.F. emessa da Sapoli.

Il fenomeno i risultato molto piiu intenso (cioe il pereento della cross-modulazione era piu alto) quanto la frequenza di modulazione era fra 1100 e $1110 \mathrm{kc} / \mathrm{s}$, cioe corrispondera a quella frequenza per cui si ha il piceo superiore della cuma a due massimi, ottenuta dall'autore, nella girointerazione nel 1949-1950 $\left(^{6}\right)$. L'altro massimo della curva di risonanza non si a potuto aceertare perche la frequenza modulante si ferma- 
va a 1250 invece che a 1400 (il secondo massimo dovrebbe esistere sulla frequenza di 1300 come si vede da Fig. 4). Oltre agli accorgimenti indicati sopra e al fatto che il massimo della percentuale di cross-modulazione si ha su 1100-1110 kc/sc, m'altra prova dell'esistenza del fenomeno e rappresentata dal fatto che il fenomeno stesso si ottiene solo e soltanto quando la riflessione dell'onda di Nola avviene intorno ai $90 \mathrm{~km}$. Il fenomeno non è stato mai notato quando la riflessione avveniva nello stirato $F$.

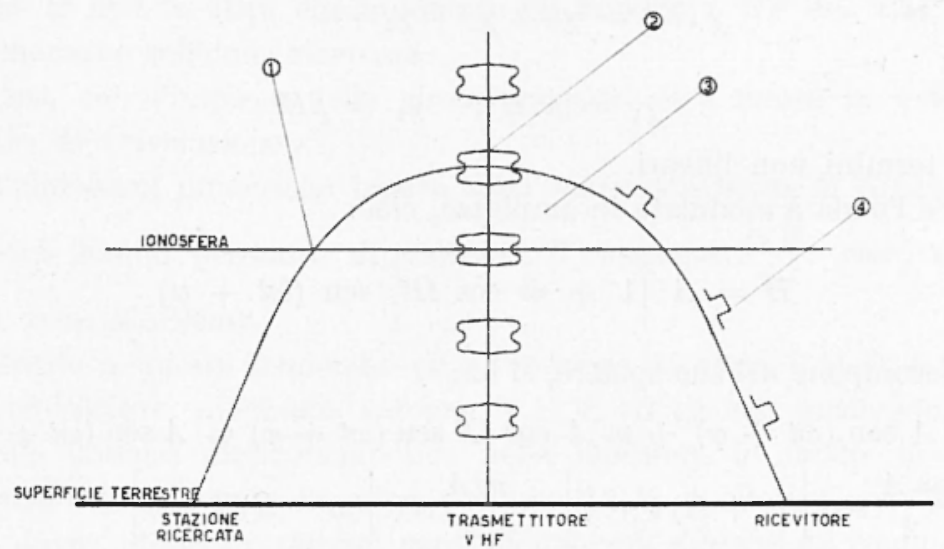

Fig. 7 - Schema rappresentante il meccanismo dell'effetto di rivelazione nella ionosfera.

1 - Onda ricercata

2 - Onda V.II.F. modulata

3 - Regione ove avviene l'effetto di rivelazione

4 - Impnlso.

Le numerose esperienze sinora compiute hanno mostrato che e possibile "liberare " da un'onda modulata l'onda di frequenza uguale alla frequenza dell'onda modulante.

In altre parole il plasma, per la sua spiceata non linearita, si comporta all'incirca come un detector. Come e stato riconosciuto da D. H. Menzel, durante un meeting tenutosi a Napoli nel 1960, l'effetto di rivelazione è completamente differente dalla cross-modulazione. L'uso dell'onda ricercata (Nola) non serve che a dimostrare che effettivamente l'inviluppo dell'onda V.H.F. modulata con frequenza pari alla girofrequenza agisce sul plasma.

Del nuovo effetto si puo dare la seguente spiegazione teorica. Questa spiegazione ci e stata suggerita dal prof. P. Caldirola. 
Se $E$ e il campo elettrico e $P$ e il vettore di polarizzazione elettrica, si ha:

$$
\bar{P}=\chi \bar{E}
$$

dove $\chi$, suscettibilita elettrica del plasma, e data da:

se il mezzo è non lineare.

$$
\chi=\chi(E)
$$

Sviluppando $\chi(E)$, si ha:

dove:

$$
\chi(E)=\chi_{0}+\chi_{1}+\chi_{2}+\ldots .
$$

$$
\chi_{1}=\alpha E ; \quad \chi_{2}=\beta E^{2}
$$

sono termini non lineari.

Se l'onda e modulata in ampiezza, cioe:

$$
E=A\lceil 1+m \cos \Omega t \mid \operatorname{sen}(\omega t+\varphi)
$$

e si decompone nel suo spettro, si ha:

$$
\begin{aligned}
E & =A \operatorname{sen}(\omega t+\varphi)+m A \cos \Omega t \operatorname{sen}(\omega t+\varphi)=A \operatorname{sen}(\omega t+\varphi)+ \\
& +\frac{m A}{2} \operatorname{sen}[(\omega+\Omega) t+\varphi]+\frac{m A}{2} \operatorname{sen}[(\omega-\Omega) t+\varphi] .
\end{aligned}
$$

Saria, per il termine quadratico:

$$
\begin{aligned}
P_{1}=\chi_{1} E=\alpha E^{2}=\alpha\{A \operatorname{sen}(\omega t+\varphi) & +\frac{m A}{2} \operatorname{sen}[(\omega+\Omega) t+\varphi]+ \\
& \left.+\frac{m A}{\underline{2}} \operatorname{sen}[(\omega-\Omega) t+\varphi]\right\} .
\end{aligned}
$$

Questa espressione contiene termini del tipo:

$$
\alpha A^{2} m \operatorname{sen}(\omega t+\varphi) \operatorname{sen}|(\omega+\Omega) t+\varphi|,
$$

e:

$$
\alpha A^{2} m \operatorname{sen}(\omega t+\varphi) \operatorname{sen}[(\omega-\Omega) t+\varphi],
$$

che sviluppati diventano, rispettivamente:

$$
\begin{aligned}
& \alpha \frac{A^{2} m}{2}\{\cos \Omega t-\cos [(2 \omega+\Omega) t+2 \varphi]\} \\
& \alpha \frac{A^{2} m}{2}\{\cos \Omega t-\cos [(2 \omega-\Omega) t+2 \varphi]\}
\end{aligned}
$$


Da qui si vede come compaia un termine in $P_{x}$ uguale a:

$$
\alpha A-m \cos \Omega t
$$

E evidente che se $\Omega$ e uguale alla girofrequenza del plasma, quest'onda verra fortemente assorbita trasferendo energia agli elettroni del mezzo. Se un'altra onda, onda ricercata, passa contemporaneamente per la stessa zona della ionosfera si genera la girointerazione. Ossia, gli impulsi di $\Omega$ (perche $\Omega$ non fa altro che modulare gli impulsi a RF del V.H.F.) si imprimeranno sull'onda ricercata.

Cosi, con l'impiego della girointerazione, si è messo in evidenza l'effetto di "rivelazione ".

Valutazioni numeriche basate sulla teoria statistica di Ginzburg e Gurevich hanno permesso di valutare il rapporto $\frac{\gamma_{1} E}{\chi_{0}}$ che risulta 20. $E$ (con $E V / \mathrm{cm})$.

Grazie a questo fenomeno ed all'impiego di onde V.H.F. e possibile concentrare, mediante antenne V.H.F. di grande guadagno, una notevole potenza elettromagnetica nella ionosfera in modo da poter eventualmente generare l'airglow previsto da V. A. Bailey nel $1938\left(^{(3)}\right.$, senza dover adoperare sistemi aerei di notevoli dimensioni come quelli che sarebbero necessari nel caso di onde medie la cui frequenza portante è proprio uguale alla girofrequenza.

\section{BIBIJOGRAFIA}

(1) MAssey H. S. W. \& BOYD R. I. F., The upper atmosphere, Edited by Hutchinson \& Co. LTD of London.

(2) Ginzburg V. L. \& Gurevicir A. V., Soviet Physics Uspekhi, 2, 201 246 (febbraio 1960); Soviet Physics Uspekhi, 3, 2, 175-284, (SettembreOttobre 1960).

(3) BaIley V. A., Phil. Mag. XXIII, 929, (1937); Phil. Mag. XXVI, 425, (1938).

(4) Cutolo M., Ricerca Scientifica e Ricostruzione, 12 (Dicembre 1946).

(5) Cutolo M., Carlevaro M. \& Ghergit M., Alta Frequenza, XV, 111, (1946).

(6) Cutolo M., Atti del Congresso Internazionale Marconiano, presso il CNR, $65,(1947)$. 
(7) Cutoso M., Il Nuovo Cimento, IX, 5, 391, (1952); Nature-London, 166, 98, (1950).

${ }^{8}$ ) Bailey V. A., SMitil R. A., Landecker K., Higgs A. I. \& Hibberd F. H., Nature-London, 169, 911 , (1952).

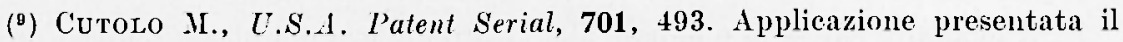
9 Dicembre 1957, concessa il 6 Maggio 1963 (in corso di pubblicazione in L.S.A.).

(10) Cu'rolo M., Nature-London, 160. 834, (1947); Alta Frequenza, XVIII, $3 \cdot 4,169(1949)$. 